\title{
Application of Monitoring Indicators in The Management of Hospital Use of Antimicrobials
}

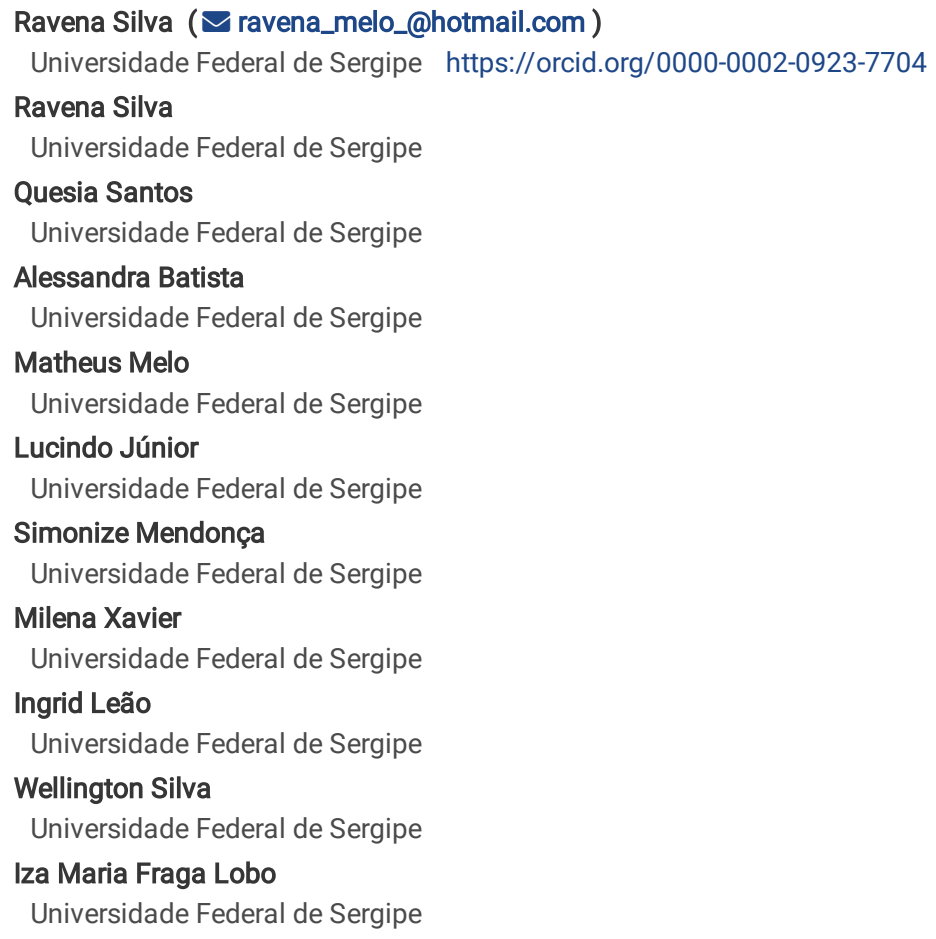

Version of Record: A version of this preprint was published at BMC Infectious Diseases on August 17th, 2021. See the published version at https://doi.org/10.1186/s12879-021-06542-5. 


\section{Abstract}

Background It is described in the literature that between $25-50 \%$ of antimicrobial prescriptions, in the hospital environment, are unnecessary or inadequate. The National Health Surveillance Agency launched the "Brazil Stewardship Project", in order to assess the national panorama of antimicrobial use management programs, therefore, it is expected that managers will be encouraged to implement the antimicrobial use management program, with publication of the results, enabling the confrontation of national data.

Methods This is an observational, analytical and prospective study conducted in a teaching hospital, with 94 active beds, distributed between the intensive care unit (ICU), the surgical clinic (SUR), the medical clinic (MED), the pneumology/infectology (PNE/INF) and pediatrics (PED).

Results During the study period, a total of 11,628 patient-days were followed up and an average of $58.3 \%$ of patients were exposed to some antimicrobial. In 376 days, for every 1,000 days of hospitalization, patients were receiving antimicrobial therapy (LOT =376/1000pd). The calculated global DOT / LOT ratio showed that each patient received an average of 1.5 antimicrobials during hospital stay.

Conclusions The indicators addressed in this study proved to be of great relevance for obtaining data regarding the use of antimicrobials, contributing to the initiatives to be taken by the Stewardship program. This study is expected to encourage other hospitals to monitor the use of antimicrobials, allowing comparison of data.

\section{Background}

One of the main public health problems worldwide is microbial resistance, a process that, although it is a natural biological phenomenon, is occurring at an accelerated rate due to the excessive use of antimicrobials (ATMs). It is described in the literature that between $25-50 \%$ of antimicrobial prescriptions, in the hospital environment, are unnecessary or inadequate, increasing hospital stay, morbidity and mortality and health care costs [1-6].

The absence of guidelines on the rational use of antimicrobials, uncertainty about the differential diagnosis, incorrect choice of medication, improper treatment duration, presence of complex comorbidities, incorrect interpretation of microbial results and lack of surveillance to monitor the use of antimicrobials are factors that imply the inappropriate use of these drugs $[5,7,8]$.

In view of the above, the Centers for Disease Control and the World Health Organization, together with the United Nations member countries, including Brazil, have been encouraging the implementation of programs such as the "Antimicrobial Stewardship Program", which is a set of interventions, audits and feedbacks, in order to optimize the use of ATMs $[2,3,9,10]$. Recently, in July 2019, the National Health Surveillance Agency launched the "Brazil Stewardship Project" [6], in order to assess the national panorama of antimicrobial use management programs, encouraging managers to implement antimicrobial use management programs, with publication of the results, enabling the confrontation of national data.

Several measures have been used to evaluate ATM use and consumption. Defined Daily Dose (DDD), which expresses consumption in grams, is the oldest and most widely used. However, alternative metrics, focused on use in days, have been proposed and adopted, such as "Days of Therapy" (DOT), equivalent to the number of days a patient receives an antimicrobial agent (regardless of the dose) and "Length of Therapy" (LOT), referring to the number of days the patient is on antimicrobial therapy (regardless of the number of drugs) $[1,9,11,12]$.

Brazil still has scarce information on the use of antimicrobials at the patient level, making it impossible to see the magnitude of the use of these drugs in this country, so this study aims to evaluate the application of the indicators DOT and LOT, in the monitoring and mapping of hospital use of antimicrobials, after the implementation of the Stewardship program in a university hospital in northeastern Brazil.

\section{Methods}

\section{Design and setting}

This is an observational, analytical and prospective study conducted in a teaching hospital, with 94 active beds, distributed between the Intensive Care Unit (ICU), the Surgical Clinic (SUR), the Medical Clinic (MED), the Pneumology/Infectology (PNE/INF) and Pediatrics (PED).

\section{Data Source}

A monthly analysis of the prescriptions of a sample of adult and pediatric patients admitted to the wards (including those who did not receive antimicrobials) was carried out between 1 January and 31 December 2018. The review of prescriptions and data extraction was performed by the researcher, using an electronic database created especially for this purpose. The data regarding the use of antimicrobials were obtained from the individual prescriptions of the patients and forms of requests for antimicrobials, under the responsibility of the hospital pharmacy.

\section{Inclusion and exclusion criteria}

Data analysis included antimicrobial agents administered intravenously or orally. The exclusion criteria involved patients with a stay of fewer than 24 hours, use of intramuscular, topical, ophthalmologic, inhaled, antiviral, and antiretroviral antimicrobials. Preoperative antimicrobial prophylaxis given in the operating room does not follow the prescription and, therefore, was excluded from the analysis.

\section{Outcome measures}


Primary outcomes were represented by the percentage of patients using antimicrobials, the number of antimicrobial prescriptions by class, and diagnostics. Secondary outcomes included analyzes of antimicrobial use, expressed in DOT, and LOT per 1000 patient-days (pd).

\section{Statistical analysis}

Data entry and storage were performed in Microsoft Excel 2019 (Microsoft Corporation, Redmond, WA, USA), transferred for statistical analysis using Stata software version 15.1 (StataCorp, College Station, Texas, USA).The variables were expressed as means and percentages. The normality of data distribution was verified and compared using the ANOVA test or the Kruskal-Wallis test with respective post-tests, as appropriate. The level of statistical significance was set at 0.05 .

\section{Results}

During the study period, a total of 11628 patient-days were followed up and an average of $58.3 \%$ of patients was exposed to some antimicrobial. The intensive care unit was the ward that contained the largest number of patients using ATM ( $86 \%$; ICU vs SUR, MED, PNE / INF, PED; $>$ < 0.05), followed by pneumology/infectology (62\%; PNE / INF vs SUR, MED; $p<0.05)$ and pediatrics (61\%; PED vs SUR, MED; $p<0.05)($ Table 1$)$.

Table 1

Distribution of antimicrobial prescriptions, by wards, 2018.

\begin{tabular}{|c|c|c|c|c|c|c|}
\hline \multirow[t]{2}{*}{ VARIABLES } & \multicolumn{6}{|l|}{ WARDS } \\
\hline & SUR & MED & PNE/INF & PED & ICU & p-value \\
\hline Use of antimicrobials & $38 \%^{\mathrm{a}}$ & $42 \% \mathrm{~b}$ & $62 \% c(a b)$ & $61 \% \mathrm{~d}(\mathrm{ab})$ & $86 \% \mathrm{e}^{(\mathrm{abcd})}$ & $0.000 *$ \\
\hline \multicolumn{7}{|l|}{ Number of prescriptions (\%) } \\
\hline First-generation cephalosporins & $49.4 \%{ }^{a(b c e)}$ & $3.8 \%{ }^{\mathrm{b}}$ & $1.4 \%^{\mathrm{c}}$ & $34.5 \% \mathrm{~d}(\mathrm{bc})$ & $9.4 \% \mathrm{e}$ & $0.000 *$ \\
\hline Fluoroquinolone & $20.5 \%^{a}$ & $14.1 \%^{\mathrm{b}}$ & $11.2 \%^{\mathrm{c}}$ & $3.6 \% \mathrm{~d}(\mathrm{abe})$ & $13.5 \%{ }^{\mathrm{e}}$ & $0.000 *$ \\
\hline Carbapenems & $1.7 \% \mathrm{a}(\mathrm{bc})$ & $11.5 \%^{\mathrm{b}}$ & $8.8 \% c$ & $6.3 \% d$ & $19.2 \% \mathrm{e}(\mathrm{abcd})$ & $0.000 *$ \\
\hline Third-generation cephalosporins & $6.5 \%^{\mathrm{a}}$ & $14.7 \% \mathrm{~b}(\mathrm{ae})$ & $8.5 \%^{c}$ & $9.2 \% \mathrm{~d}$ & $4 \%^{\mathrm{e}}$ & $0.004^{\star}$ \\
\hline Metronidazole & $10.1 \%^{\mathrm{a}}$ & $5.8 \% \mathrm{~b}$ & $1.7 \% \mathrm{c}(\mathrm{ae})$ & $6.3 \% d$ & $11.1 \% \mathrm{e}^{\mathrm{e}}$ & $0.031^{*}$ \\
\hline Cefepime & $0.8 \%^{a}$ & $10.3 \% \mathrm{~b}(\mathrm{a})$ & $15.6 \%$ (ade) & $6.0 \% d$ & $7.1 \% \mathrm{e}$ & $0.000 *$ \\
\hline Clindamycin & $9.1 \%$ & $7.1 \%$ & $5.1 \%$ & $2.1 \%$ & $7.4 \%$ & 0.072 \\
\hline Antifungals & $0.2 \%^{\mathrm{a}}$ & $7.1 \% \mathrm{~b}(\mathrm{a})$ & $16 \% \mathrm{c}(\mathrm{ad})$ & $4.5 \%{ }^{d}$ & $6.7 \% \mathrm{e}^{\mathrm{e}}$ & $0.000 *$ \\
\hline Glycopeptides & $1.1 \%^{\mathrm{a}}$ & $6.4 \% \mathrm{~b}$ & $3.4 \%^{\mathrm{c}}$ & $3 \% d$ & $10.4 \% \mathrm{e}^{\mathrm{e}(\mathrm{acd})}$ & $0.001 *$ \\
\hline Azithromycin & $0 \%^{\mathrm{a}}$ & $2.6 \% \mathrm{~b}$ & $9.5 \% \mathrm{c}(\mathrm{abe})$ & $6.3^{\% d(a e)}$ & $1.7 \%^{\mathrm{e}}$ & $0.000 *$ \\
\hline Penicillins & $0.2 \%^{\mathrm{a}}$ & $10.3 \% \mathrm{~b}(\mathrm{ae})$ & $3.4 \%^{c}$ & $8.3 \% \mathrm{~d}(\mathrm{ae})$ & $1 \%^{\mathrm{e}}$ & $0.000 *$ \\
\hline Sulfamethoxazole + trimethoprim & $0 \%^{\mathrm{a}}$ & $3.2 \% \mathrm{~b}$ & $10.9 \%$ (abe) & $3.6 \% \mathrm{~d}(\mathrm{a})$ & $3 \%{ }^{\mathrm{e}}$ & $0.000 *$ \\
\hline Aminoglycosides & $0.4 \%^{\mathrm{a}}$ & $2.6 \% \mathrm{~b}$ & $4.4 \%^{c}$ & $6.5 \% \mathrm{~d}(\mathrm{a})$ & $3.4 \%^{\mathrm{e}}$ & $0.025^{\star}$ \\
\hline Polymyxin B & $0 \%^{\mathrm{a}}$ & $0.6 \% \mathrm{~b}$ & $0 \% c$ & $0 \% d$ & $2 \% \mathrm{e}(\mathrm{acd})$ & $0.014^{\star}$ \\
\hline \multicolumn{7}{|c|}{ Subtitle: SUR - Surgical Clinic, MED - Medical Clinic, PNE/INF - Pneumology / Infectology, PED - Pediatrics, ICU - Intensive Care Unit. ${ }^{\star} p<0.05$} \\
\hline
\end{tabular}

The 1st generation cephalosporins were the most prescribed classes both in surgical clinic (49.9\%; SUR vs MED, PNE / INF, ICU; $p<0.05)$ and in pediatrics (34.5\%; PED vs MED, PNE / INF; $p$ < 0.05). The medical clinic had a higher number of prescriptions referring to 3rd generation cephalosporins (14.7\%; MED vs SUR, ICU; $p<0.05)$, pneumology/infectology had a higher number of antifungals ( $16 \%$; PNE / INF vs SUR, PED; $p<0.05)$ and the ICU prevailed the prescription of carbapenems (19.2\%; ICU vs SUR, MED, PNE / INF, PED; $p<0.05)$ (Table 1).

Exposure time to 1st generation cephalosporins in the surgical clinic (123 DOT / 1000pd; SUR vs MED, PNE / INF; p < 0.05) and in pediatrics (94 DOT / 1000pd; PED vs MED, PNE / INF; $p<0.05$ ) was superior to the other antimicrobial classes. Higher exposures were also observed for quinolone in the medical clinic (57 DOT / 1000pd), sulfamethoxazole + trimethoprim in pneumology/infectology (121 DOT / 1000pd) and carbapenems in the ICU (368 DOT / $1000 \mathrm{pd}$; ICU vs SUR, MED, PED; $p<0.05$ ) (Table 2). 
Table 2

Distribution of use rates for antimicrobial classes, by wards, 2018 .

\begin{tabular}{|c|c|c|c|c|c|c|}
\hline \multirow[t]{2}{*}{ VARIABLES } & \multicolumn{6}{|l|}{ WARDS } \\
\hline & SUR & MED & PNE/INF & PED & ICU & p-value \\
\hline \multicolumn{7}{|l|}{ DOT/1000pd } \\
\hline Fluoroquinolone & $108^{a}$ & $57^{b}$ & $71^{\mathrm{c}}$ & $24^{d}$ & $147^{\mathrm{e}(\mathrm{bd})}$ & $0.001^{\star}$ \\
\hline First-generation cephalosporins & $123^{a(b c)}$ & $5^{b}$ & $6^{c}$ & $94^{\mathrm{d}(\mathrm{bc})}$ & $73^{\mathrm{e}(\mathrm{bc})}$ & $0.000 *$ \\
\hline Carbapenems & $11^{\mathrm{a}}$ & $43^{b}$ & $84^{\mathrm{c}(a)}$ & $40^{d}$ & $368^{e(a b d)}$ & $0.000 *$ \\
\hline Third-generation cephalosporins & 43 & 50 & 64 & 61 & 35 & 0.775 \\
\hline Cefepime & $8^{\mathrm{a}(\mathrm{ce})}$ & $32^{\mathrm{b}}$ & $95^{\mathrm{c}}$ & $47^{d}$ & $114^{\mathrm{e}}$ & $0.000 *$ \\
\hline Metronidazole & $56^{\mathrm{a}}$ & $12^{\mathrm{b}}$ & $8^{c(a d)}$ & $59^{d}$ & $119^{\mathrm{e}(\mathrm{bc})}$ & $0.000 *$ \\
\hline Antifungals & $1^{\mathrm{a}(\mathrm{ce})}$ & $24^{\mathrm{b}}$ & $83^{c}$ & $40^{d}$ & $118^{\mathrm{e}}$ & $0.000 *$ \\
\hline Sulfamethoxazole + trimethoprim & $0^{\mathrm{a}(\mathrm{cde})}$ & $4^{b(c)}$ & $121^{\mathrm{c}}$ & $25^{d}$ & $72^{\mathrm{e}}$ & $0.000 *$ \\
\hline Clindamycin & 47 & 28 & 37 & 12 & 75 & 0.099 \\
\hline Glycopeptides & $8^{a}$ & $25^{\mathrm{b}}$ & $36^{c}$ & $10^{d}$ & $183^{e(a b c d)}$ & $0.000^{*}$ \\
\hline Aminoglycosides & 6 & 16 & 32 & 34 & 83 & 0.055 \\
\hline Penicillins & $1^{\mathrm{a}(\mathrm{bd})}$ & $35^{\mathrm{b}}$ & $18^{\mathrm{c}}$ & $38^{d}$ & $13^{\mathrm{e}(\mathrm{bd})}$ & $0.000 *$ \\
\hline Azithromycin & $0^{a(c d)}$ & $8^{\mathrm{b}(\mathrm{c})}$ & $40^{c}$ & $31^{d}$ & $23^{\mathrm{e}}$ & $0.000 *$ \\
\hline Polymyxin B & $0^{\mathrm{a}}$ & $5^{\mathrm{b}}$ & $0^{c}$ & $0^{d}$ & $59^{e(a c d)}$ & 0.010 \\
\hline LOT/1000pd & $300^{a}$ & $262^{b}$ & $470^{c(a b)}$ & $382^{d}$ & $753^{e(a b d)}$ & $0.000 *$ \\
\hline DOT/LOT ratio & $1.4^{\mathrm{a}}$ & $1.3^{\mathrm{b}}$ & $1.5^{\mathrm{c}}$ & $1.4^{\mathrm{d}}$ & $2.0^{\mathrm{e}(\mathrm{abcd})}$ & $0.000 *$ \\
\hline \multicolumn{7}{|c|}{ Subtitle: SUR - Surgical Clinic, MED - Medical Clinic, PNE/INF - Pneumology / Infectology, PED - Pediatrics, ICU - Intensive Care Unit. *p < 0.05} \\
\hline
\end{tabular}

In 376 days, for every 1000 days of hospitalization, patients were receiving antimicrobial therapy (LOT=376/1000pd). Comparing hospital wards, the ICU was where patients were exposed to ATMs for the longest time (753 DOT / 1000pd; ICU vs SUR, MED, PED; $p<0.05$ ), followed by pneumology/infectology (470 DOT / 1000pd; PNE / INF vs SUR, MED; $p<0.05$ ). The calculated global DOT / LOT ratio showed that each patient received an average of 1.5 antimicrobials during hospital stay, with a higher number within the ICU (2 DOT / LOT; ICU vs SUR, MED, PNE / INF, PED; p < 0.05) (Table 2).

Surgical prophylaxis was the main diagnostic for the use of antimicrobials in the surgical clinic (80.8\%; SUR vs MED, PNE / INF; $p<0.05)$ and in pediatrics (36.2\%; PED vs MED, PNE / INF; p < 0.05). Respiratory infections were higher in the medical clinic (22.4\%) and pneumology/infectology (39.1\%; PNE / INF vs SUR; $p<0.05)$, while in the ICU the diagnosis of sepsis prevailed $(34.1 \%$; ICU vs SUR; $p<0.05)$. There was no significant difference in the diagnosis of skin / soft tissue infections, urinary infections, abdominal infections and others (Table 3 ).

Table 3

Distribution of diagnostic for the use of antimicrobials, by hospital ward, in 2018.

\begin{tabular}{|c|c|c|c|c|c|c|}
\hline \multirow[t]{2}{*}{ DIAGNOSTIC } & \multicolumn{6}{|l|}{ WARDS } \\
\hline & SUR & MED & PNE/INF & PED & ICU & p-value \\
\hline Surgical prophylactic & $80.8 \% \mathrm{a}(\mathrm{bc})$ & $0 \% \mathrm{~b}$ & $0 \%^{\mathrm{c}}$ & $36.2 \% \mathrm{~d}^{(\mathrm{bc})}$ & $33.9 \% \mathrm{e}$ & $0.000 *$ \\
\hline Respiratory infection & $4.2 \% \mathrm{a}$ & $22.4 \%^{b}$ & $39.1 \% \mathrm{c}(\mathrm{a})$ & $32.9 \%^{d}$ & $19.8 \% \mathrm{e}^{\mathrm{e}}$ & $0.017 *$ \\
\hline Sepsis & $0 \% \mathrm{a}$ & $19.0 \%^{b}$ & $19.6 \%^{\mathrm{c}}$ & $5.4 \%$ d & $34.1 \% \mathrm{e}(\mathrm{a})$ & $0.033^{*}$ \\
\hline Skin/soft tissue infection & $2.5 \%$ & $17.2 \%$ & $16.3 \%$ & $10.1 \%$ & $0.9 \%$ & 0.154 \\
\hline Urinary infection & $2.5 \%$ & $19.0 \%$ & $14.1 \%$ & $5.4 \%$ & $3.8 \%$ & 0.749 \\
\hline Abdominal infection & $7.5 \%$ & $19.0 \%$ & $1.1 \%$ & $0.7 \%$ & $7.5 \%$ & 0.134 \\
\hline Others & $2.5 \%$ & $3.4 \%$ & $9.8 \%$ & $9.4 \%$ & $0 \%$ & 0.141 \\
\hline
\end{tabular}

Subtitle: SUR - Surgical Clinic, MED - Medical Clinic, PNE/INF - Pneumology / Infectology, PED - Pediatrics, ICU - Intensive Care Unit. 
Others - Surgical Site Infection, Visceral Leishmaniasis, Febrile Neutropenia, Bacterial Endocarditis. * $p<0.05$.

$a, b, c, d, e-$ statistical differences per line (Bonfferroni $p<0.05)$

\section{Discussion}

The present study found that an average of $58.3 \%$ of patients was exposed to some antimicrobial. A large study carried out by Versporten [7], when evaluating the use of antimicrobials in patients admitted to adult hospitals in 53 countries, identified, in Latin America, the average use of $36.8 \%$, ranging from $32.5-$ 43.4\%. However, the literature describes a broader range that extends from $22-76 \%$ of hospitalized patients receiving at least one antimicrobial during hospital stay $[1,4,8,9,12]$. Even though the result, observed here, is within the presented range, these studies demonstrate variations that may be related to the type of care provided in each hospital, where the need for antimicrobial indication may be higher or lower.

The greatest number of patients exposed to the use of antimicrobials was in the intensive care unit ( $86 \%)$, followed by pneumology/infectology (62\%) and pediatrics (61\%). The predominance of the use of ATMs in the ICU was also observed in a study of one-off prevalence on health-related infections in Austria, in which $67.9 \%$ of patients in this ward were exposed to some antimicrobial, other studies indicate variations from $55.1-57 \%[1,4,7]$. Variations are also observed in the medical clinic (31.8\% - 73.2\%), and in the surgical clinic (37.3-84.1\%) $[1,7,8,13]$. The weak conditions with which patients arrive at the ICUs explain the greater frequency of infections and, consequently, the greater need for antibiotic therapy in this ward.

The 1st generation cephalosporins were the most prescribed classes and had longer use time at the surgical clinic (49.9\%; 123 DOT / 1000pd) and in pediatrics (34.5\%; 94 DOT / 1000pd); the medical clinic had a higher number of prescriptions referring to 3rd generation cephalosporins (14.7\%), however the greatest exposure time was for quinolones (57 DOT / 1000pd); pneumology/infectology predominated the use of antifungals (16\%) and longer use for sulfamethoxazole + trimethoprim (121 DOT / 1000pd). In the ICU, carbapenems (19.2\%; 368 DOT / 1000pd) stood out both in the number of prescriptions and the time of use.

The use of 1 st generation cephalosporins and quinolones in the hospital under study is mainly related to the extension of prophylaxis in surgeries, which requires a more critical look at their real need, considering that in early January 2019, European Medicines Agency released a report recommending the suspension and restriction of the use of some antibiotics, among them quinolones and fluoroquinolones, because of the serious, disabling and potentially permanent side effects that these drugs have presented. The literature also reports a significant correlation between the use of cephalosporins and fluoroquinolones with the increase in carbapenem-resistant Enterobacteriaceae [14, 15].

A study conducted by Kimura [16], which evaluated the long-term effects of antibiotic administration programs at a university hospital in Japan, found that therapy days per 1000 patient-days were higher for 1 st generation cephalosporins (45 DOT / 1000pd). The time of use of quinolones (4.7 DOT / 1000 pd) and carbapenems (13.5 DOT / 1000pd) were well below the values verified in this study. However, studies show results ranging from 25.8 DOT / 1000 pd to 132.3 DOT / 1000pd for quinolones and 8.7 DOT / 1000pd to 39.1 DOT / 1000pd for carbapenems [2, 9, 10], this variation may be related to the lack of uniformity in data collection, which implies the importance of standardizing the obtainment of these rates, allowing reliable comparability of the data.

In 376 days, for every 1000 days of hospitalization, patients were receiving antimicrobial therapy (LOT = 376/1000pd), with the longest time observed in the ICU (753 DOT / 1000pd), followed by pneumology/infectology (470 DOT / 1000pd). The LOT indicator is rarely found in the literature to express the use of antimicrobials, however, a study in the United States of America found an average LOT of 536 (median, 529; range, 427-684) per 1000 patient-days in 70 American hospitals [12], a value above that observed in this study. In addition to the gap in publications on the different monitoring measures for antimicrobial management programs, the lack of disclosure of monitoring strategies and measures in hospitals in low and middle-income countries stands out. A recent meta-analysis of 221 studies using clinical trials, interrupted time series, and other methods evaluated the effectiveness of antibiotic management programs, in which unfortunately few studies represent these countries [17].

The ICU and pneumology/infectology presented LOTs well above the overall hospital rate, reflecting the severity and frequency of community and hospital infections in patients assisted in these two wards, which require more frequent, intense, and longer antimicrobial therapy. The calculated global DOT / LOT ratio showed that each patient received an average of 1.5 antimicrobials during the hospital stay, with a higher number prescribed within the ICU (2 DOT / LOT). The literature points to similar values ranging from 1.3 to 2.1 antimicrobials per patient $[4,5,8]$.

The surgical clinic and pediatrics pointed out surgical prophylaxis as the main diagnostic for the use of antimicrobials (80.8\% and $36.2 \%$, respectively), which is in line with the greater number of indications of 1 st generation cephalosporins in these wards. Highlights for the prophylactic use of antimicrobials, as observed in this study, were reported by Talaam [5], in a rural hospital in western Kenya, where prophylaxis was responsible for $56.3 \%$ of antimicrobial indications. Gutema [8], documented that the prophylactic use of antimicrobials represented $41.3 \%$ in the prescriptions in the surgical clinic and $8.5 \%$ in the medical clinic.

Respiratory infections prevailed in the medical clinic and pneumology/infectology ( $22.4 \%$ and $39.1 \%$, respectively), this data reflects the characteristics of the wards described, and corroborate with studies that describe that approximately $50 \%$ of the use of antimicrobials is used in the treatment of respiratory and urinary infections $[4,8,9,13]$. In the ICU the main diagnostic indications were for the treatment of sepsis (34.1\%). Sepsis is a serious public health problem in ICUs in Brazil, this pathology is the second leading cause of mortality within this environment. Empirical treatment with antibiotics is usually initiated with broad-spectrum drugs, such as carbapenems, observed in this study, possibly due to the number of multidrug-resistant strains isolated in these patients [18].

The strengths of this study include the prospective design with direct observation of the prescriptions, allowing a greater precision of the analysis regarding the use of antimicrobials. However, some limitations were noted. First, the study was conducted at a single center. Second, comorbidities have not been 
evaluated. Third, the adequacy of the prescriptions was not addressed. Fourth, no data were obtained on the prevalence of bacterial pathogens and their susceptibility patterns. Fifth, the analysis of temporal trends has not been carried out.

\section{Conclusion}

The indicators addressed in this study proved to be of great relevance for obtaining data regarding the use of antimicrobials, contributing to the initiatives to be taken by the Stewardship program, recently implemented in the hospital, to improve patient care and safety. All data found were in accordance with the ranges observed in the literature, which implies the accuracy of the information. This study is expected to encourage other hospitals to monitor the use of antimicrobials, allowing comparison of data.

\section{Abbreviations}

ATM

Antimicrobials

DDD

Defined Daily Dose

DOT

Days of Therapy

ICU

Intensive Care Unit

LOT

Length of Therapy

MED

Medical Clinic

PED

Pediatrics

PD

Patient-days

PNE/INF

Pneumology/Infectology

SUR

Surgical Clinic

\section{Declarations}

Ethics approval and consent to participate. This study was approved by the Research Ethics Committee of the Federal University of Sergipe, with CAAE: 74181317.8.0000.5546, under opinion No. 2,256.79. A waiver of informed consent was obtained due to the non-interventional nature of this study and the complete anonymity of patient data.

Consent for publication. Not applicable.

Availability of data and materials. All data generated or analyzed during this study are included in this published article [and its supplementary information files].

Competing interests. The authors declare that they have no competing interests.

Funding. Not applicable.

Authors' contributions. RMRS collected, analyzed, and interpreted the data and wrote the work. QNS, AMB, MSM, and INL helped with data collection. LJQJ, SCBM, and MMX reviewed and collaborated on the work structure. WBS, and IMFL contributed to the conception of the work and substantially revised it. All authors read and approved the final manuscript.

Acknowledgments. Not applicable.

\section{References}

1. Nathwani D, editor. Antimicrobial stewardship: from principles to practice. British Society for Antimicrobial Chemotherapy. http://www.bsac.org.uk/antimicrobialstewardshipebook/BSAC-AntimicrobialStewardship-FromPrinciplestoPractice-eBook.pdf. Published 2018.

2. Morrill HJ, Caffrey AR, Gaitanis MM, LaPlante KL. Impact of a prospective audit and feedback antimicrobial stewardship program at a veterans affairs medical center: A six-point assessment. PLoS One. 2016. doi:10.1371/journal.pone.0150795.

3. Agência Nacional De Vigilância Sanitária (ANVISA). Diretriz nacional para elaboração de programa de gerenciamento do uso de antimicrobianos em serviços de saúde. 
http://portal.anvisa.gov.br/documents/33852/271855/Diretriz+Nacional+para+Elabora\%C3\%A7\%C3\%A3o+de+Programa+de+Gerenciamento+do+Uso+de 7edc-411b-a7e0-49a6448880d4. Published 2017.

4. Segagni LL, Blacky A, Starzengruber P, Diab-Elschahawi M, Wrba T, Presterl E. A national point prevalence study on healthcare-associated infections and antimicrobial use in Austria. Wien Klin Wochenschr. 2016. doi:10.1007/s00508-015-0947-8.

5. Talaam RC, Abungana MM, Ooko PB. An antibiotic audit of the surgical department at a rural hospital in Western Kenya. Pan Afr Med J. 2018. doi:10.11604/pamj.2018.29.219.14510.

6. Agência Nacional De Vigilância Sanitária (ANVISA). Projeto stewardship brasil. Avaliação nacional dos programas de gerenciamento do uso de antimicrobianos em unidade de terapia intensiva adulto dos hospitais brasileiros.

http://portal.anvisa.gov.br/documents/33852/271855/Projeto+Stewardship+Brasil/435012dc-4709-4796-ba78-a0235895d901. Published 2019.

7. Versporten A, Zarb P, Caniaux I, Gros MF, Drapier N, Miller M, Jarlier V, Nathwani D, Goossens H. Antimicrobial consumption and resistance in adult hospital inpatients in 53 countries: results of an internet-based global point prevalence survey. Lancet Glob Heal. 2018. doi:10.1016/S2214109X(18)30186-4.

8. Gutema G, Håkonsen H, Engidawork E, Toverud EL. Multiple challenges of antibiotic use in a large hospital in Ethiopia - A ward-specific study showing high rates of hospital-acquired infections and ineffective prophylaxis. BMC Health Serv Res. 2018. doi:10.1186/s12913-018-3107-9.

9. Baggs J, Fridkin SK, Pollack LA, Srinivasan A, Jernigan JA. Estimating national trends in inpatient antibiotic use among US hospitals from 2006 to 2012. JAMA Intern Med. 2016. doi:10.1001/jamainternmed.2016.5651.

10. Dubrovskaya Y, Scipione MR, Siegfried J, Jen SP, Pham V, Papadopoulos J, Decano A, Lewis T, Dabestani A. Multilayer model of pharmacy participation in the antimicrobial stewardship program at a large academic medical center. Hosp Pharm. 2017. doi:10.1177/0018578717726993.

11. Antimicrobial Stewardship Programs (ASPS). - Metrics Examples (Public Health Ontario). https://www.publichealthontario.ca/en/eRepository/ASP_Metrics_Examples.pdf. Published 2017.

12. Polk RE, Hohmann SF, Medvedev S, Ibrahim O. Benchmarking risk-adjusted adult antibacterial drug use in 70 US academic medical center hospitals. Clin Infect Dis. 2011. doi:10.1093/cid/cir672.

13. Fortini A, Faraone A, Di Pietro M, Cappugi C, Magnante G, Boccadori C, Bartolini S, Rabatti L. Antimicrobial stewardship in an Internal Medicine ward: effects on antibiotic consumption and on the use of carbapenems. Intern Emerg Med. 2018. doi:10.1007/s11739-018-1916-9.

14. European Medicines Agency (EMA)

Human medicines: highlights of 2018

Published 2019.

European Medicines Agency (EMA). Human medicines: highlights of 2018. https://www.ema.europa.eu/en/documents/report/human-medicineshighlights-2018_en.pdf. Published 2019.

15. European Centre for Disease Prevention and Control (ECDC). Rapid risk assessment: Carbapenem-resistant Enterobacteriaceae - first update. Stockholm: ECDC. https://ecdc.europa.eu/sites/portal/files/documents/RRA-Enterobacteriaceae-Carbapenems-European-Union-countries.pdf. Published 2018.

16. Kimura T, Uda A, Sakaue T, Yamashita K, Nishioka T, Nishimura S, Ebisawa K, Nagata M, Ohji G, Nakamura T, Koike C, Kusuki M, loroi T, Mukai A, Abe Y, Yoshida H, Hirai M, Arakawa S, Yano I, Iwata K. Tokimatsu. Long-term efficacy of comprehensive multidisciplinary antibiotic stewardship programs centered on weekly prospective audit and feedback. Infection. 2018. doi:10.1007/s15010-017-1099-8.

17. Davey P, Marwick CA, Scott CL, Charani E, McNeil K, Brown E, Gould IM, Ramsay CR. Michie. Interventions to improve antibiotic prescribing practices for hospital inpatients. Cochrane Database Syst Rev. 2013. doi:10.1002/14651858.CD003543.pub3.

18. Barros LLS, Maia CSF, Monteiro MC. Fatores de risco associados ao agravamento de sepse em pacientes em Unidade de Terapia Intensiva. Cad saúde colet. 2016. doi:10.1590/1414-462x201600040091. 